\title{
Erratum to: Mechanical endovascular thrombectomy for acute ischemic stroke: a retrospective multicenter study in Belgium
}

\author{
Niels Fockaert ${ }^{1} \cdot$ Marieke Coninckx $^{2} \cdot$ Sam Heye $^{3} \cdot$ Luc Defreyne $^{4} \cdot$ \\ Denis Brisbois $^{5} \cdot$ Pierre Goffette $^{6} \cdot$ Jan Gralla $^{7} \cdot$ Pasquale Mordasini $^{7}$. \\ Andre Peeters $^{8} \cdot$ Philippe Desfontaines $^{9} \cdot$ Dimitri Hemelsoet $^{2} \cdot$ Vincent Thijs $^{1,10,11}$. \\ Robin Lemmens ${ }^{1,10,11}$
}

Published online: 4 November 2015

(C) Belgian Neurological Society 2015

\section{Erratum to: Acta Neurol Belg \\ DOI 10.1007/s13760-015-0552-7}

In the online version of the original article, Figs. 1 and 2 captions have been erroneously switched. The correct version of the figures and their captions are given in the following page.

The online version of the original article can be found under doi:10.1007/s13760-015-0552-7.

Robin Lemmens

robin.lemmens@uzleuven.be

1 Department of Neurology, University Hospitals Leuven, 3000 Leuven, Belgium

2 Department of Neurology, University Hospitals Gent, 9000 Ghent, Belgium

3 Department of Radiology, University Hospitals Leuven, 3000 Leuven, Belgium

4 Department of Vascular and Interventional Radiology, University Hospitals Gent, 9000 Ghent, Belgium

5 Department of Interventional Neuroradiology, Centre Hospitalier Chrétien, 4000 Liège, Belgium

6 Department of Neuroradiology, Cliniques Universitaires Saint-Luc, Université catholique de Louvain, Brussels, Belgium
7 Department of Diagnostic and Interventional Neuroradiology, University Hospital Bern, Bern, Switzerland

8 Department of Neurology, Cliniques Universitaires SaintLuc, Université catholique de Louvain, 1200 Brussels, Belgium

9 Department of Neurology, Centre Hospitalier Chrétien, 4000 Liège, Belgium

10 Department of Neurosciences, Experimental Neurology and Leuven Research Institute for Neuroscience and Disease (LIND), KU Leuven, University of Leuven, 3000 Leuven, Belgium

11 VIB, Vesalius Research Center, Laboratory of Neurobiology, 3000 Leuven, Belgium 
Fig. 1 Distribution of postprocedure mTICI scores, stratified by site of AOL. Substantial reperfusion, defined as an mTICI 2B-3, was achieved in $78 \%$ of patients in the overall patient series; in $73 \%$ with an occlusion in the anterior circulation versus $100 \%$ with an posterior circulation occlusion $(p=0.02)$. ICA internal carotid artery, $M C A$ medial cerebral artery, $B A$ basilar artery, $A O L$ arterial occlusive lesion, $m$ TICI modified thrombolysis in cerebral infarction

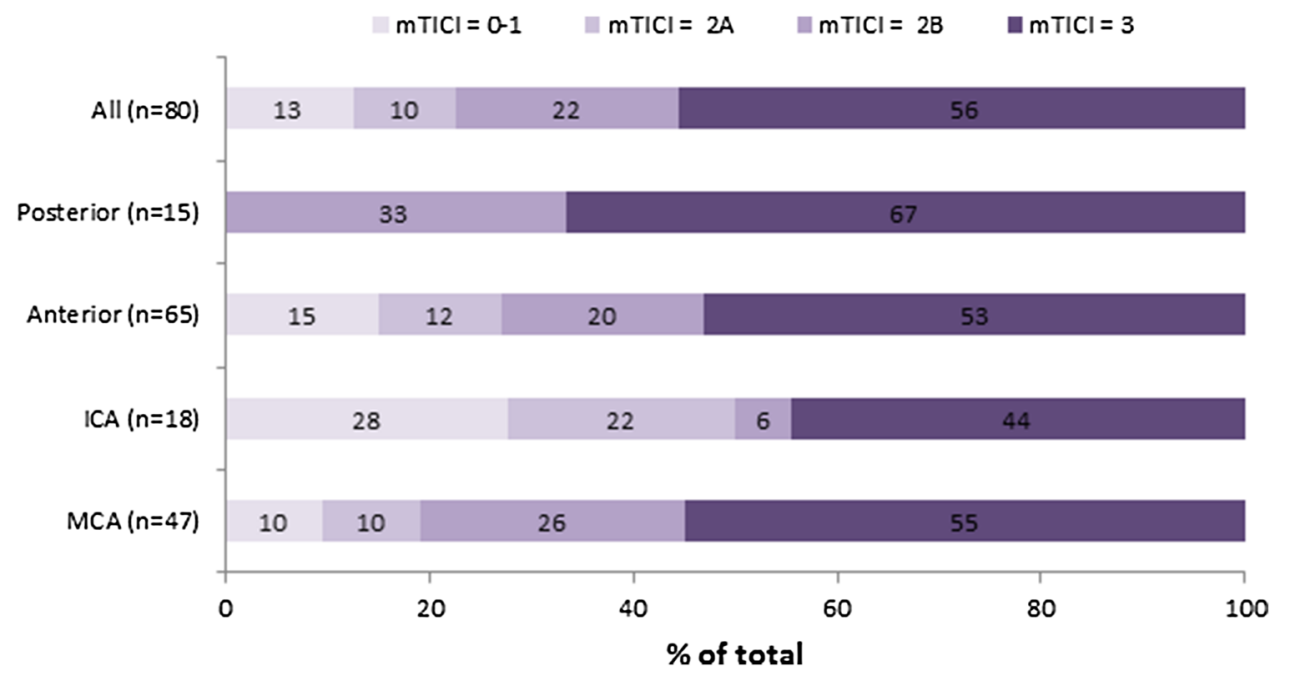

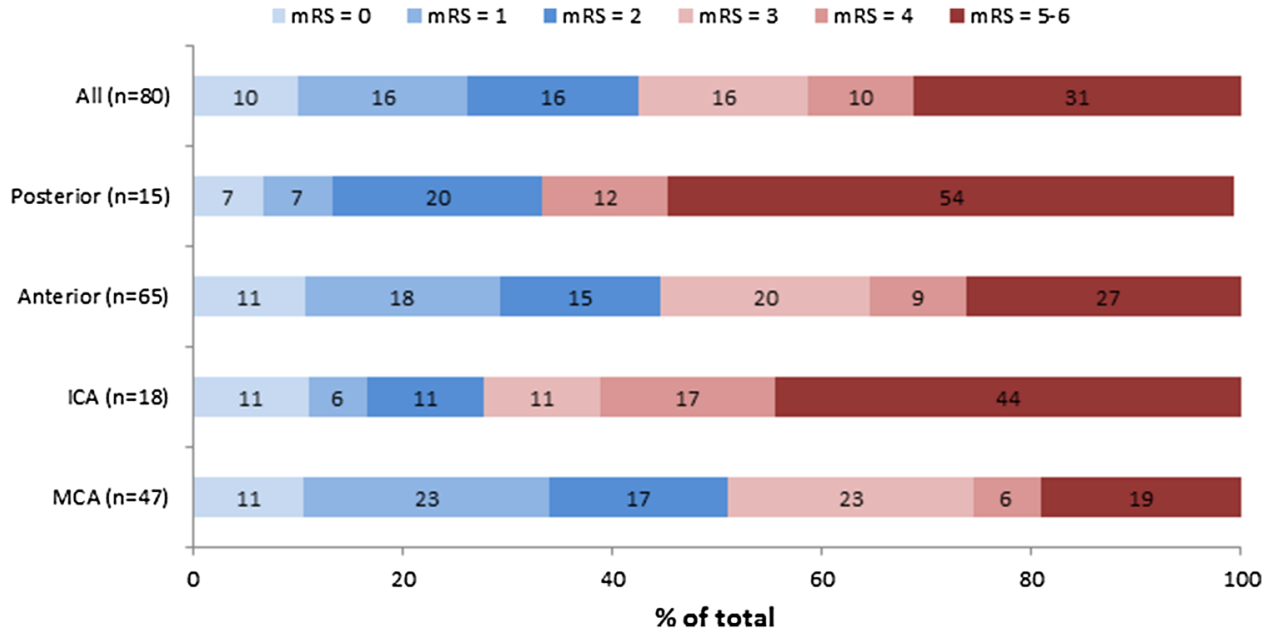

Fig. 2 Functional outcome in all patients, stratified by AOL. In the overall population, GFO was achieved in $42 \%$ of patients and EFO in $26 \%$ EFO. When stratified by anterior versus posterior circulation stroke, no differences were observed: The rate of GFO was $44 \%$ in patients with anterior circulation stroke versus $34 \%$ in patients with basilar artery stroke. ICA internal carotid artery, $M C A$ medial cerebral artery, $B A$ basilar artery, $m R S$ modified Rankin Scale, $G F O$ good functional outcome (mRS 0-2), EFO excellent functional outcome (mRS 0-1) 